\title{
Effects of the Moon on the Earth in the Past, Present, and Future
}

\author{
Rina Rast*, Sarah Finney, Lucas Cheng, Joland Schmidt, \\ Kessa Gerein, \& Alexandra Miller ${ }^{\dagger}$
}

\begin{abstract}
The Moon has fascinated human civilization for millennia. Exploration of the lunar surface played a pioneering role in space exploration, epitomizing the heights to which modern science could bring mankind. In the decades since then, human interest in the Moon has dwindled. Despite this fact, the Moon continues to affect the Earth in ways that seldom receive adequate recognition. This paper examines the ways in which our natural satellite is responsible for the tides, and also produces a stabilizing effect on Earth's rotational axis. In addition, phenomena such as lunar phases, eclipses and lunar libration will be explained. While investigating the Moon's effects on the Earth in the past and present, it is hoped that human interest in it will be revitalized as it continues to shape life on our blue planet.
\end{abstract}

Keywords: Moon, Earth, tides, Earth's axis, lunar phases, eclipses, seasons, lunar libration

\section{Introduction}

Decades have come and gone since humans' fascination with the Moon galvanized exploration of the galaxy and observation of distant stars. During the Space Race of the 1950 and 1960 , nations strived and succeeded in reaching Earth's natural satellite to propel mankind to new heights. Although this interest has dwindled and humans have largely distanced themselves from the Moon's relevance, its effects on Earth remain as far-reaching as ever. Not only is our rocky satellite responsible for the tides, which are part of the very rhythm and heartbeat of life on our planet, but it also affects the Earth's rotational axis, and makes conditions more favourable for life itself. Human interest in the Moon has also been fueled by other phenomena such as lunar phases, eclipses and libration. In recognizing and propagating these effects, the goal of this study is to rekindle the fascination humans once held for the Moon and space exploration in general.

\section{Four Prominent Lunar Effects on Earth Effect on Oceans and Tides}

When we think of the relationship between the Earth and the Moon, perhaps the first thing that comes to mind is the tides. Since the dawn of our existence, the regular rise and fall of the water levels along our shores have continued to symbolize the Moon's unceasing influence on our planet. They have contributed to making

*Department of Physics, College of Arts and Science, University of Saskatchewan, Saskatoon, SK, Canada

Correspondence: kgrog9@mail.usask.ca

†This paper was written through the joint effort of all authors. 


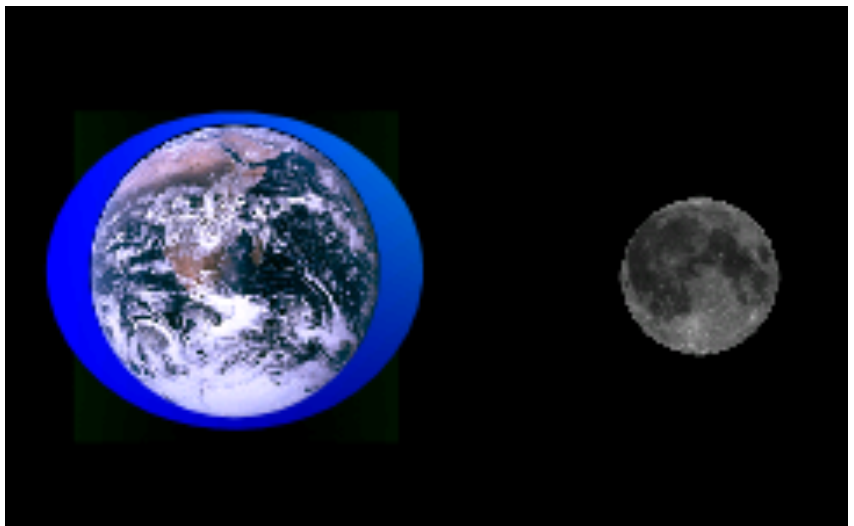

Figure 1 - Inertia and gravitation work together to cause the tides.

Source: https://commons.wikimedia.org/wiki/File:Tide S2.PNG

Permission: CC-BY-SA 3.0

Author: Theresa Knott

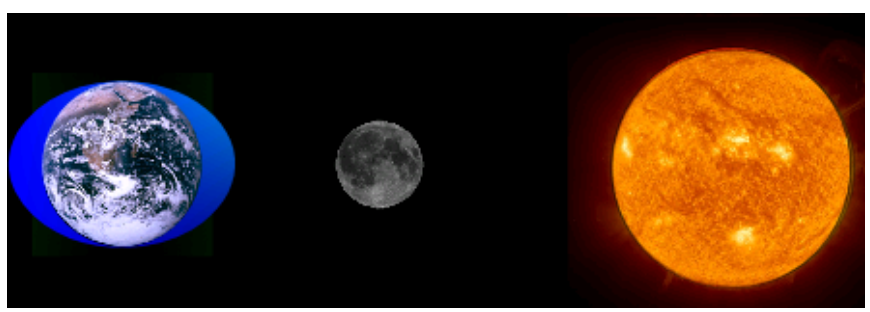

Figure 2 - Spring tides occur when the Sun's gravitational pull adds to that of the Moon.

Source: https://commons. wikimedia.org/wiki/File:Tide S3.PNG

Permission: CC-BY-SA 3.0

Author: Theresa Knott

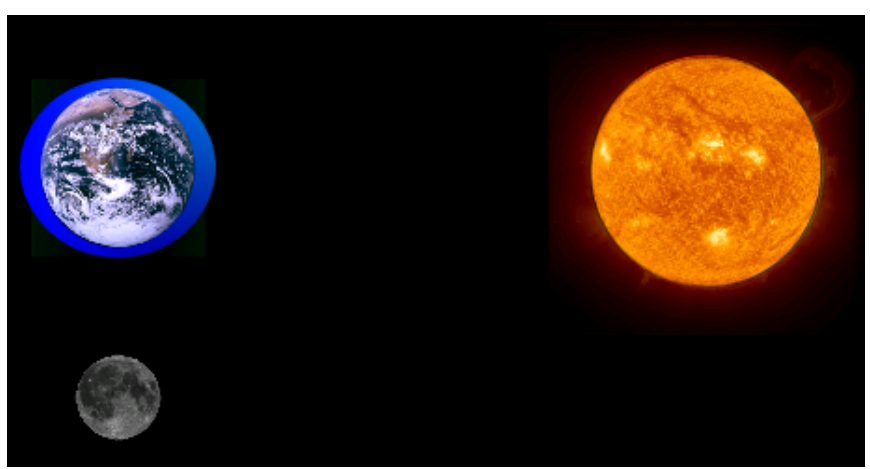

Figure 3 - Neap tides occur when the gravitational pulls of the Moon and Sun counteract each other. Source: https://commons.wikimedia.org/wiki/File:Tide S4.PNG

Permission: CC-BY-SA 3.0

Author: Theresa Knott conditions here more favourable to life, and are caused mainly by the combination of two things: gravity and inertia. ${ }^{1}$ The ocean tides create an environment unique in our Solar System, where life can experience both immersion in water and exposure to air within a time interval of only a few hours. In this way, organisms are briefly exposed to the stresses of a foreign world, and then returned to their original habitat. It is possible that these changes could help promote the move from one environment to the other. ${ }^{2}$ For this reason, it seems entirely plausible that the Moon's existence could have triggered the movement of Earth's aquatic life onto land. ${ }^{2}$

But what causes tides? Roughly four hundred years ago, Galileo believed the up and down movement of the sea level was analogous to water sloshing around in a vase. ${ }^{3}$ Later, Newton explained the phenomenon using a concept he himself had developed: gravitation. ${ }^{4}$ Today, we consider Newton's explanation to be the correct one.

The Moon exerts a gravitational force on the Earth. Although its mass is only approximately $1 / 100$ th the mass of the Earth, 1 the force it exerts on the Earth is strong enough to cause the oceans-which are obviously less dense than the continents-to bulge towards the moon. They follow it as the Earth rotates beneath them. ${ }^{1}$ In addition to this pull of gravity, inertia plays a role in creating the tides. Inertia is defined as an object's tendency to resist changes in motion. ${ }^{5}$ As the water on the side of the Earth that is closest to the moon is experiencing a pull from the Moon, the water farthest from it is experiencing the least amount of gravity, and therefore it remains unmoved. This creates two bulges on either side of the Earth, explaining why there are two high tides and two low tides at a given location every day. ${ }^{6}$ Since the Earth is not a perfect sphere, and the continental masses obstruct the oceans, the difference between high and low tides varies from location to location. ${ }^{1}$

The difference in height between high and low tides varies as the Moon waxes and wanes, with the largest range occurring once per month. ${ }^{6}$ Around each new and full moon, the Sun, Earth and Moon are more or less aligned in space. At these times, the gravitational pull of the Sun adds to that of the Moon. The average solar tide is roughly $50 \%$ of the average lunar tide. For this reason, the tide is at its maximum range at new moon or full moon. We call this the spring tide-not named after the season, but rather in the sense of jumping, rising, or bursting forth, since this is the most extreme tide. ${ }^{6}$

The smallest tides are called neap tides, and they occur when the Sun's gravity is counteracting the Moon's. ${ }^{6}$ These take place roughly twice per month, around the first quarter and third quarter phases. Therefore, there is approximately a 7-day interval between spring tides and neap tides. ${ }^{6}$ 


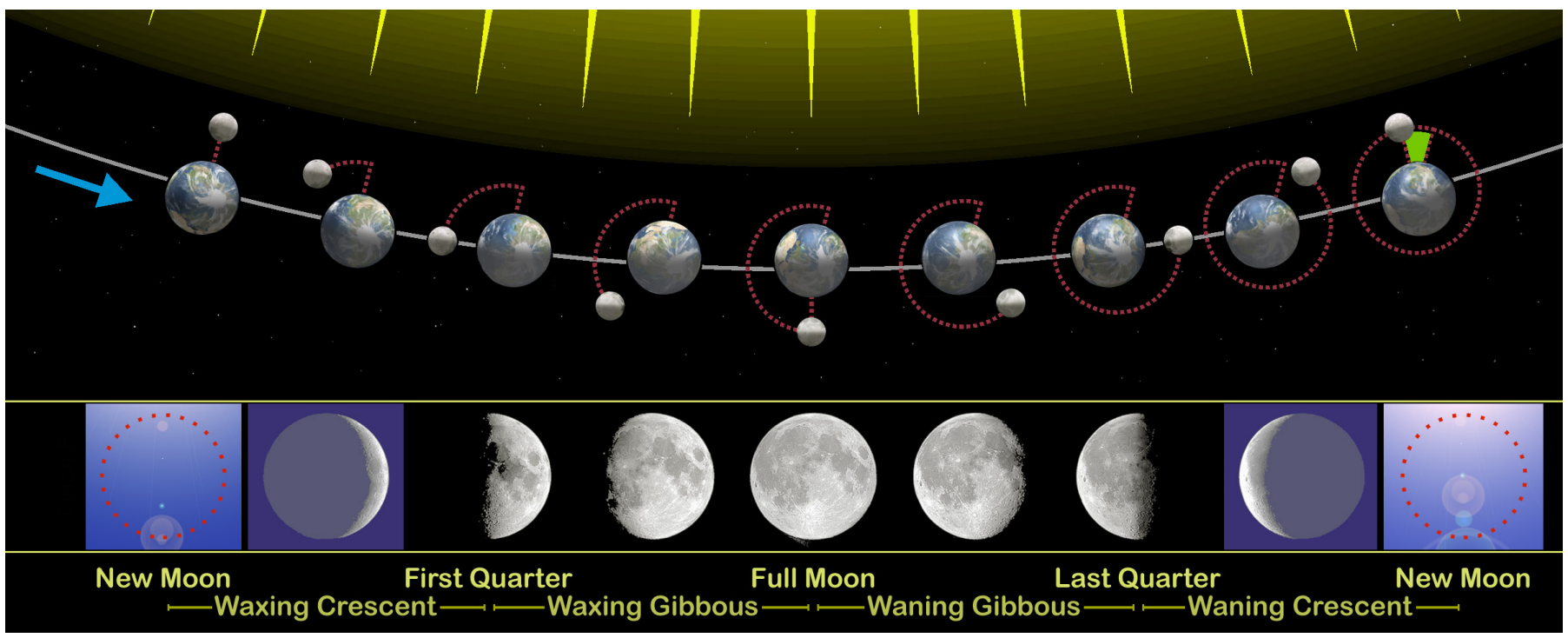

Figure 4 - Phases of the Moon.

Source: https://commons.wikimedia.org/wiki/File:Moon_phases_en.jpg

Permission: CC-BY-SA 3.0

Author: Orion 8

\section{Effect of Different Lunar Phases}

The relative positions of the Sun, Moon and Earth do much more than influence our tides. They are also responsible for the phases that our rocky satellite exhibits, ${ }^{7}$ which have already been briefly mentioned. This phenomenon is something most people are quite familiar with: over the course of roughly a month, the face of the Moon that is turned toward Earth goes from being completely dark, to fully illuminated, and then back to completely dark again. This smooth fluctuation can be explained by the changing angles between the Sun, Moon and Earth.

Let's start our journey through the Moon's phases at new Moon. This occurs when the Sun, Moon and Earth are in a straight line, with the Moon in the middle. Therefore, from the vantage point of Earth, the Moon is so close to the Sun that it cannot be discerned at all. As the Moon orbits Earth, it moves eastward in the sky, lagging behind the Sun. As this happens, it becomes visible in the evening sky and observers on Earth will see more of the sunlit side of the Moon with each successive night. This begins as a waxing crescent, and matures to a first quarter when the Moon's disc is half illuminated. As you can see in the diagram, the Moon is at a $90^{\circ}$ angle with respect to the Earth and Sun at this point. ${ }^{7}$ The following nights, the Moon's surface is more than half illuminated, and it is called a waxing gibbous. Finally, half-way through the lunar cycle, the Moon is aligned with the Sun and Earth-but instead of being in the middle, as it is at new Moon, the Earth is in the middle. At this time, the Moon is fully illuminated and we say that it is full. It rises as the sun sets, remains in the sky throughout the entire night, and sets at sunrise. ${ }^{8}$

Immediately after full Moon, the process described above seems to start working in reverse. The Moon appears to grow thinner each night, and it is called a waning gibbous. When it reaches a $90^{\circ}$ angle with respect to the Earth and Sun once again, it is only half illuminated and we say that it has reached its third quarter phase. At this point, the portion of the disc that was dark at first quarter phase is now lit. After reaching third quarter, the Moon becomes a waning crescent until it reaches new Moon phase once again.

\section{Effect on Earth's Rotational Axis}

To further discuss the remaining lunar-earth phenomena, it is important to also comprehend the effects the Moon has on the rotational axis of the Earth. The Moon provides stability to the Earth's axial tilt and other rotational functions, which is vital to Earthly phenomena like the seasons that will be discussed in-depth in the next section.

First, it is necessary to consider how the Earth obtained its axial tilt. There have been multiple theories proposed to explain the axial tilt of the earth, however the Giant Impact Hypothesis has the most traction and scientific support. ${ }^{9}$ In this hypothesis, it is theorized that a large impact between two planet-sized objects occurred. One object was known as Theia, while the other object was a young and newly forming planet that would become 


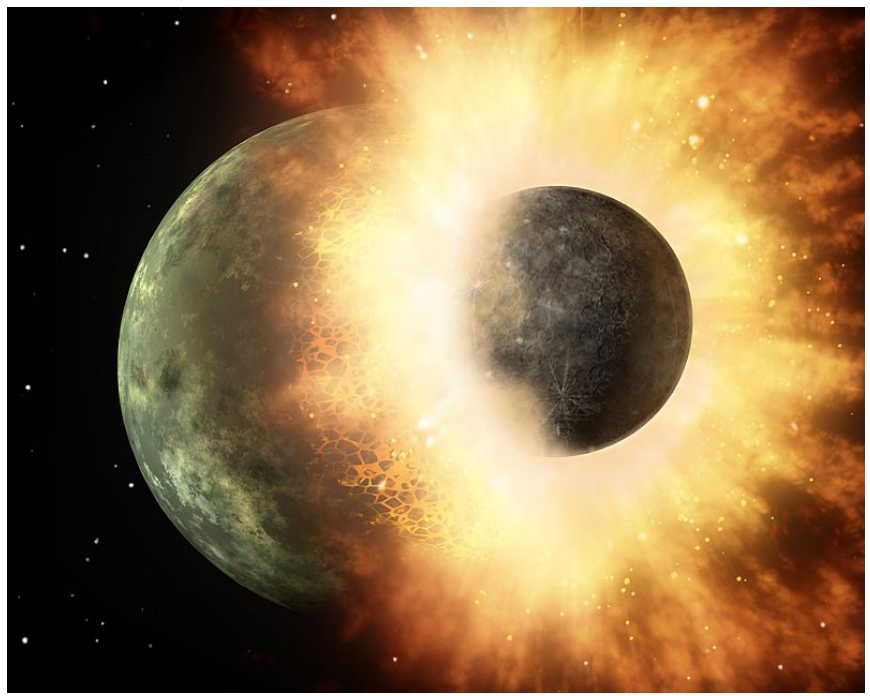

Figure 5 - Artist's Conception of a Collision Between Two Bodies.

The moon may have formed as a result of a similar collision.

Source: https://en.wikipedia.org/wiki/Giantimpact_hypothesis\#/media/File:Artist\%27s_concept_o f_collision_at_HD_172555.jpg

Permission: Public domain

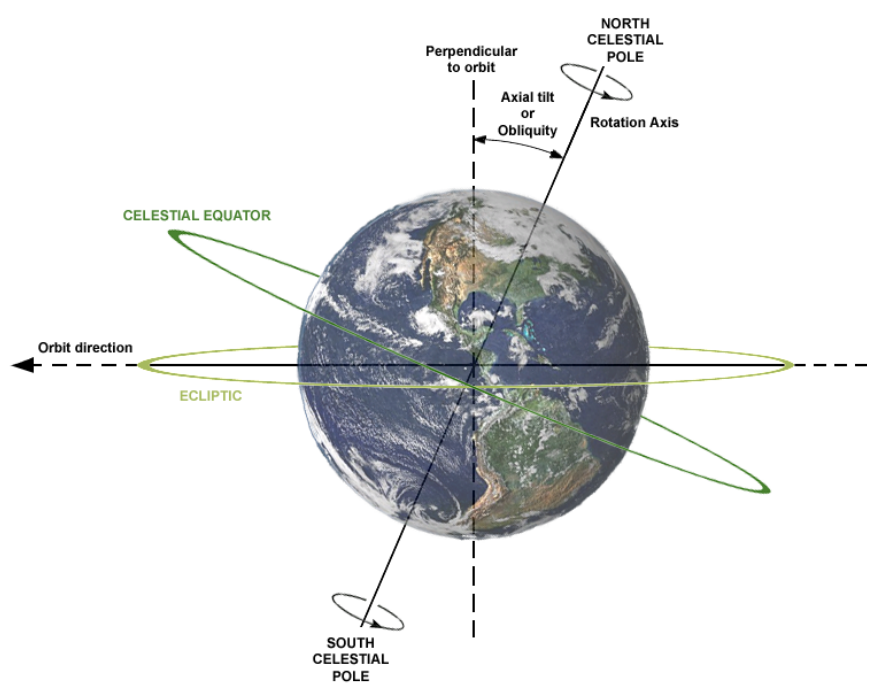

Figure 6 - The Axial Tilt of the Earth In Respect to the Plane of the Ecliptic

Source:

https://commons.wikimedia.org/wiki/File:AxialTiltObli quity.png

Permission: CC-BY-SA 3.0

Author: Dennis Nilsson
Earth. As a result of the collision, Theia's debris along with other debris from the impact coalesced together to form the Moon, while the impact left the Earth with a tilted axis. ${ }^{9}$ Therefore, the Moon has not only provided stability to the Earth's tilt since its formation, but may also be a significant contributor to the existence of the tilt itself.

As for the resulting impact on the Earth's orientation, the current " $23.5^{\circ}$ tilt of the Earth's axis of rotation is a relic of the oblique collision that [also] produced the Moon." ${ }^{2}$ The magnitude of the Earth's tilt is not constant, varying from $22.1^{\circ}$ to $24.5^{\circ}$ over a complete cycle of approximately 41,000 years. ${ }^{10}$ In simpler terms, the Earth is always amidst this cycle, but due to the small variation of $2.4^{\circ}$ and the long period of the tilt's fluctuation sequence, the effects are hard to recognize without longterm statistical analysis. This is where the Moon deserves credit on various levels. As discussed in the oceans and tides section, the Moon exerts strong gravitational forces on the Earth, but aside from affecting waves and oceans, the pull "may [also be] key to making Earth a livable planet by moderating the degree of wobble in Earth's axial tilt." ${ }^{11}$ Without this moderating force, "astronomers have predicted that Earth's tilt could vary as much as $85^{\circ} . .^{12}$ It then becomes clear the Moon provides substantial stability and moderation, as without it, there is close to a 35 times $\left(2.4^{\circ}\right.$ variation compared to a $85^{\circ}$ variation) increase in variation. This variation would not be conducive to life sustaining activities around the Earth and most evidently weather patterns would change drastically and sporadically without this stability. ${ }^{12}$

The gravitational force that stabilizes the axial tilt of the Earth also has an effect on the precession of the Earth's axis. A precession is the circular, cone-shaped, wobble that exists with most rotating objects, and it occurs when the rotating orientation of the tilted object changes through excessive twisting.13 In this case it is the Moon's gravitational force along with other gravitational forces in the solar system (most pertinently, from the Sun) induce these wobbles in the Earth's axial rotation.10 Similar to the cycle of Earth's axial tilt, the natural precession cycle is around 26,000 years.10 Therefore, in a theoretical sense, the combination of changes in both precession and tilt may have severe effects on the Earth-Sun radiation transfer budget, but due to the relatively long cycles of both of these processes, the real effects that they have on the Earth are hard to quantify in the short term. In the long-term, these two aforementioned cycles, along with the eccentricity cycle of the Earth, can be grouped together and classified as components of the Milankovitch Cycles. The Milankovitch Cycles are the collective results from these three rotational cycles that influence the long-term climatic patterns on Earth.10 The Milankovitch Cycles are more 


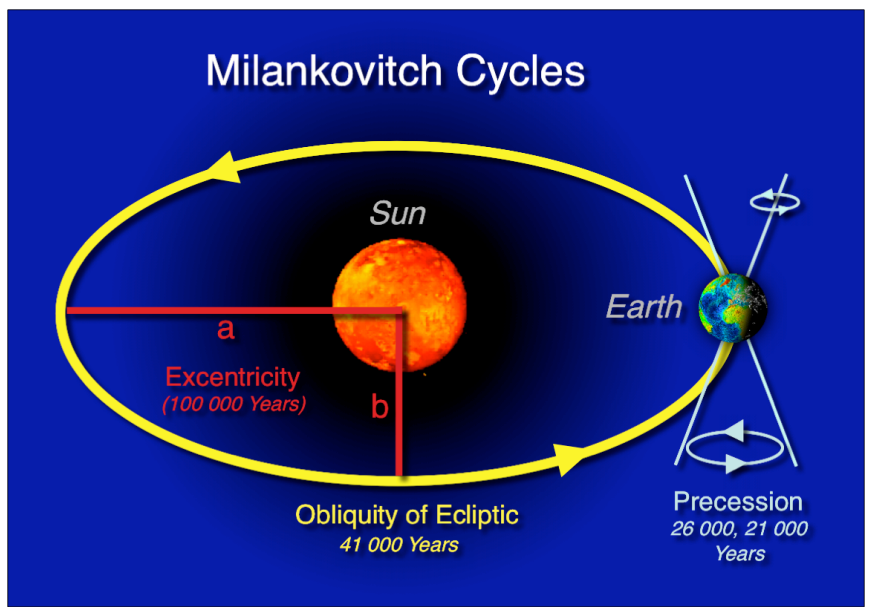

Figure 7 - The Milankovitch Cycles: Eccentricity and Precession

Source: https://commons.wikimedia.org/wiki/File:

Milankovitch-cycles_hg.png

Permission: CC-BY-SA 2.5

Author: Hannes Grobe, Alfred Wegener Institute for

Polar and Marine Research

related to the Earth's movement around the Sun, but the Moon plays a vital role in moderating these cycles and thus is mentioned to disclose and support the Moon's importance in these fundamental Earth-Sun rotational processes.

\section{Effect on Earth's Seasons}

As mentioned above, one of the vital functions resulting from the angle and stability of Earth's axial tilt is the formation of seasons. If you have ever wondered why summer, winter, or any season occurs when it does, where it does, further analyzing the effect of the Earth's tilt on climate and the Moon's contribution to those changes in maintaining its stability is essential.

As we now know, the Earth is currently tilted at about $23.5^{\circ}$ on its ecliptic plane. ${ }^{2}$ This means at certain points in the Earth's orbit around the sun, either the Northern hemisphere, Southern hemisphere, or the equator (twice in one full orbit) receives the highest levels of insolation; which is solar energy that reaches the Earth's surface. This occurs when each respective location is angled by the Earth's tilt more directly towards the Sun. ${ }^{14}$ These instances when the Sun is directly overhead can be easily identified throughout the year, by the length of day, the weather experienced, and are better known as the solstices and equinoxes. Solstices transpire in the northern and southern hemisphere when the longest and shortest days occur. The Summer solstice is the longest day (June $21^{\text {st }}$ in the Northern Hemisphere and December $21^{\text {st }}$ in the Southern Hemisphere), while the Winter solstice is the shortest day (December $21^{\text {st }}$ in the Northern Hemisphere and June $21^{\text {st }}$ in the Southern Hemisphere). Equinoxes occur when the equator receives the most direct sunlight, and length of day and night are relatively equal. ${ }^{14}$ These dates and terms are important, as it provides us where the Earth is in its orbit, and helps to reaffirm that it is indeed tilted on its axis. Comparably, "if the Earth's was at right angles to the plane of its orbit around the Sun, there would be no solstice days and no seasons [experienced]. ${ }^{\prime \prime 15}$

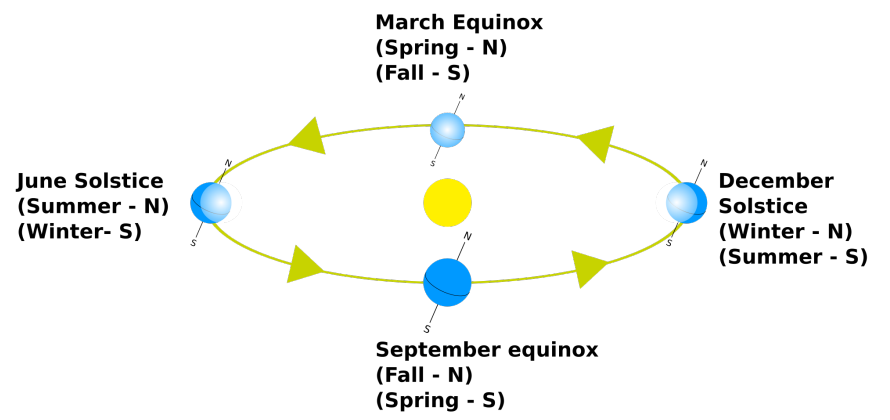

Figure 8 - The Solstices and Equinoxes of the Earth over a Full Orbit

Source: https://commons.wikimedia.org/wiki/File:

Orbital_relations_of_the_Solstice,_Equinox_\%26_Inte rvening_Seasons.svg

Permission: Public Domain

Low density of

incident rays

(northern winter)

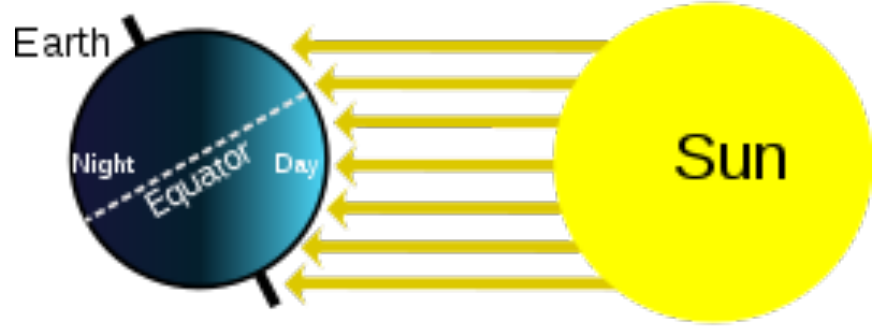

$$
\begin{gathered}
\text { High density of } \\
\text { incident rays } \\
\text { (southern summer) }
\end{gathered}
$$

Figure 9 - How Axial Tilt Affects Intensity of Energy Received

Source: https://en.wikipedia.org/wiki/Effect_of_Sun_ angle_on_climate\#/media/File:Seasons.svg

Permission: Public domain

In addition to manifestation of seasons, recognizing the aforementioned stabilization effects of the Moon on the Earth in this discussion is equally important. As the Moon orbits around the Earth, it creates a constant force along the transverse plane of Earth's axis. This additional stabilizing force requires an even more substantial impact or aberration for changes to occur in the rotational axis and tilt of the Earth. ${ }^{16}$ It is the angle of the 
Earth's tilt that creates the necessary conditions for Earth to experience seasons, but it is the Moon's stabilizing ability in combination with other climate moderation functions (like the ocean and atmosphere) that has allowed for these life sustaining conditions to persist for a relatively long period of time. ${ }^{11}$

To gain a better understanding of the Earth's tilt and its effect on the seasons, a couple examples in both extremes will help to understand the effects different tilt angles may have. Examining the figure presented below, we can see at $0^{\circ}$ tilt, the poles receive little to no energy, while the equator receives most, if not all, solar radiation. In such a case we expect equatorial regions to experience much warmer weather and potentially too hot of weather, while regions close to the poles experience converse situations. We can see with the next few angles, as they increase $\left(22.5^{\circ}\right.$ to $45^{\circ}$ to $\left.60^{\circ}\right)$ the poles increasingly face the sun more directly, and as a result began to receive increasing levels of solar radiation. The polar circles even enlarge with increasing size of angles and thus lead to eventually nightless days at the poles. At the full $90^{\circ}$ tilt, only the poles receive direct solar radiation and the equator would become highly inhabitable due to climate.

This exemplification of different tilt angles further illustrates the necessity of the Moon in moderating our Earth's tilt, as without it, our Earth would be subjected to continuous fluctuations in tilt angle and in turn climate. Thus, never settling at an angle that is advantageous to sustaining life. Ultimately, Earth's seasons are dependent on several aspects of its orbit, but the tilt of the Earth and the Moon's ability to maintain that tilt is essential to our opportune climate.

\section{Other Interesting Lunar-Earth Phenomena}

\section{Lunar Libration}

Despite the fact that the Moon is tidally locked to Earth and experiences synchronous rotation, observers on our planet can actually see roughly $59 \%$ of the Moon's surface from Earth. ${ }^{17}$ This is caused by a combination of effects known as lunar librations. "Libration" comes from the Latin for "balance scales, ${ }^{18}$ and it refers to the Moon's appearance of "wobbling" back and forth. There are three kinds: libration in latitude, libration in longitude, and diurnal libration. ${ }^{17}$

Libration in latitude occurs because the Moon's axis is inclined relative to Earth. From our vantage point, our view of the Moon fluctuates between peeking over its north and south poles, giving the impression of the Moon slowly nodding its head over the course of the lunar month. Libration of longitude occurs because the Moon does not orbit with constant speed-it travels slower when it is farther away from the Earth, and faster when it is close. Finally, libration can also be observed over the course of a single night-a phenomenon known as diurnal libration. When the Moon rises, the its western edge is facing the Earth. As it sets, an observer's viewpoint has shifted to up to four thousand miles away to the east. This makes causes the Moon to appear as if it's shaking its head "no" over the course of the night. ${ }^{17}$

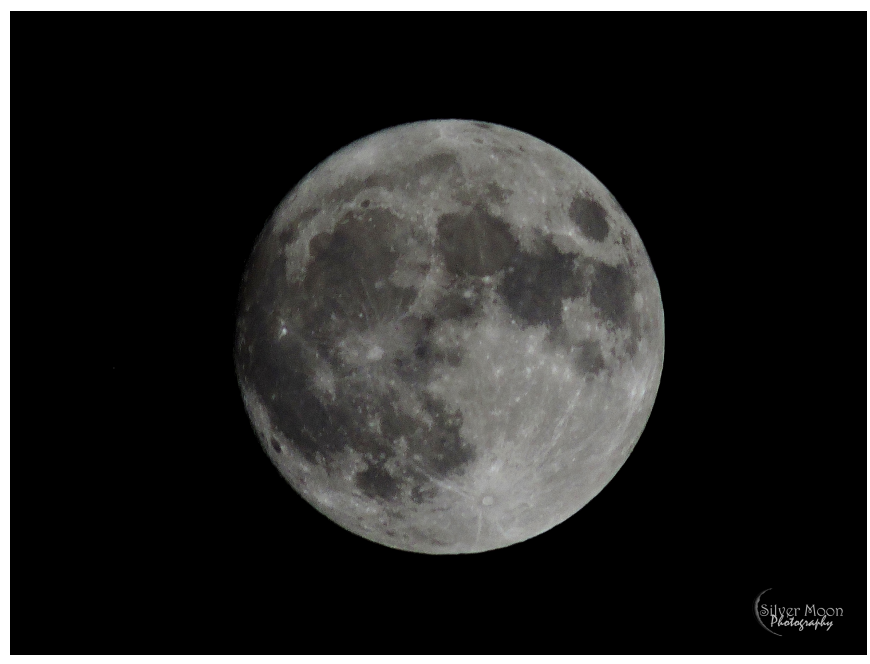

Figure 10A - Example of diurnal libration. Photograph taken at 9:30 PM on 13 November 2016. Compare to Figure 10B.

Photography Credit: Rina Rast, Silver Moon

Photography

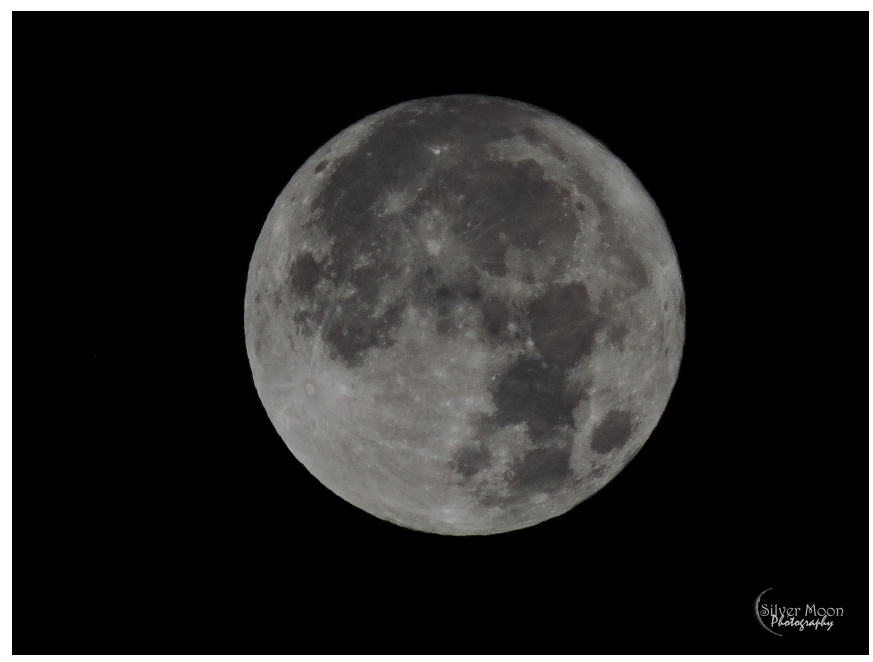

Figure 10B - Example of diurnal libration. Photograph taken at 5:30 AM on 14 November 2016. Note how the craters and lunar maria have "moved" relative to Figure $10 \mathrm{~A}$.

Photography Credit: Rina Rast, Silver Moon 


\section{Eclipses}

Many of us have had the opportunity to watch as the full Moon is slowly gobbled up by a dark shadow, then turns an eerie copper red, and finally creeps out from under the ominous shadow to appear like an ordinary full moon once again. A smaller number of lucky individuals have had the chance to observe the Sun becoming dark in the middle of a clear day, plunging the world into inky darkness. These events must have seemed very alarming to the ancient civilizations who were strongly in tune with the motions and patterns of heavenly bodies. Today, we call them eclipses. They are some of the most interesting astronomical events on a sky-watcher's calendar, and they are made possible through the alignment of the Sun, Moon and the Earth.

Eclipses of the Moon, also called lunar eclipses, occur when the Sun, Moon and Earth are aligned, with the Earth in the middle. They can only occur at full Moon, as our satellite passes through a portion of Earth's shadow. This shadow is actually made up of two cone-shaped shadows, called the umbra and penumbra. ${ }^{19}$ The umbral shadow is the zone where all of the Sun's light is blocked by the Earth. The umbral shadow is the zone where all of the Sun's light is blocked by the Earth. The penumbral shadow, which encircles the umbra, is the region in which the Earth blocks only part of the Sun's rays from reaching the Moon. ${ }^{19}$

If the Moon passes through the Earth's penumbra, penumbral eclipses occur. These represent about $35 \%$ of all eclipses, ${ }^{18}$ but they are difficult to observe and are only of academic interest, as Earth's outer shadow is very hard to discern on the surface of the Moon. Another $30 \%$ are called partial eclipses, occurring when only part of the Earth's umbral shadow strikes the Moon. ${ }^{19}$ The remaining $35 \%$ of eclipses are by far the most awe-inspiring. These occur when the entire Moon passes through the Earth's umbra, and are called total eclipses. They can last for more than 6 hours, ${ }^{20}$ and they are famous for the copper-red colour that the Moon takes on during totality.

Eclipses of the Sun, or solar eclipses, occur when the Moon passes between the Earth and the Sun, at new Moon. If the three objects are aligned properly, the Moon's shadow falls on the Earth's surface and observers can see some portion of the Sun's light being blocked by the Moon. ${ }^{20}$ Like the Earth, the Moon has two nested shadows as well-the penumbra and the umbra. When a region of the Earth passes through the Moon's penumbra, observers in that region see a partial solar eclipse. When the Moon's umbral (or inner) shadow crosses the Earth, a total solar eclipse is seen. Sometimes, when the Moon is on the far side of its orbit, it appears too small on Earth to fully block the light from the Sun. When this occurs, observers see a ring of light around the Moon, and an annular eclipse occurs.

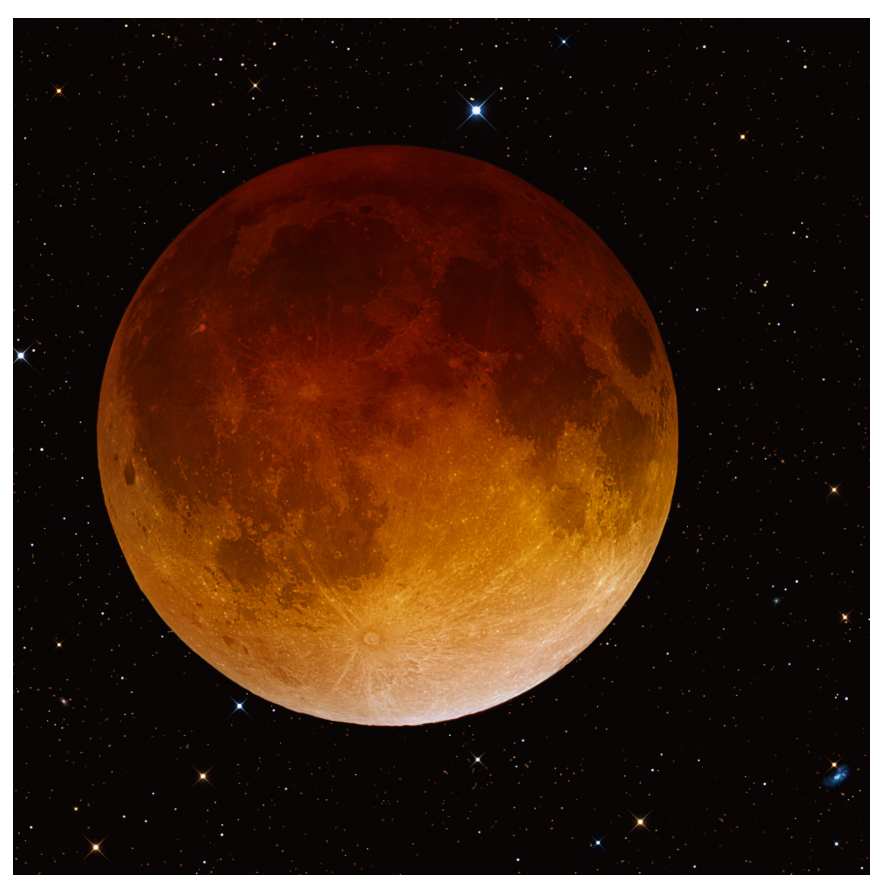

Figure 11 - Total Lunar Eclipse, April 15, 2014 Source: https://commons.wikimedia.org/wiki/File:Lunar _eclipse_04-15-2014_by_R_Jay_GaBany.jpg

Permission: CC-BY-SA 3.0

Author: Robert Jay GaBany

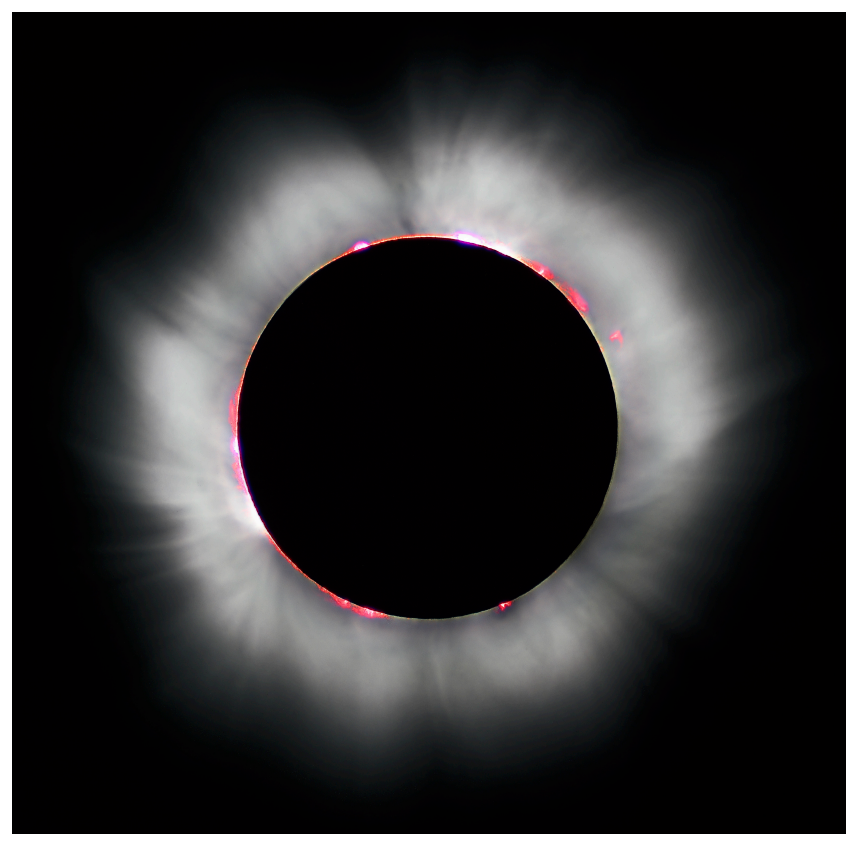

Figure 12 - Total Solar Eclipse, 1999

Source: https://commons.wikimedia.org/wiki/File:Solar _eclipse_1999_4.jpg

Permission: CC BY-SA 3.0

Author: Luc Viatour / www.Lucnix.be 


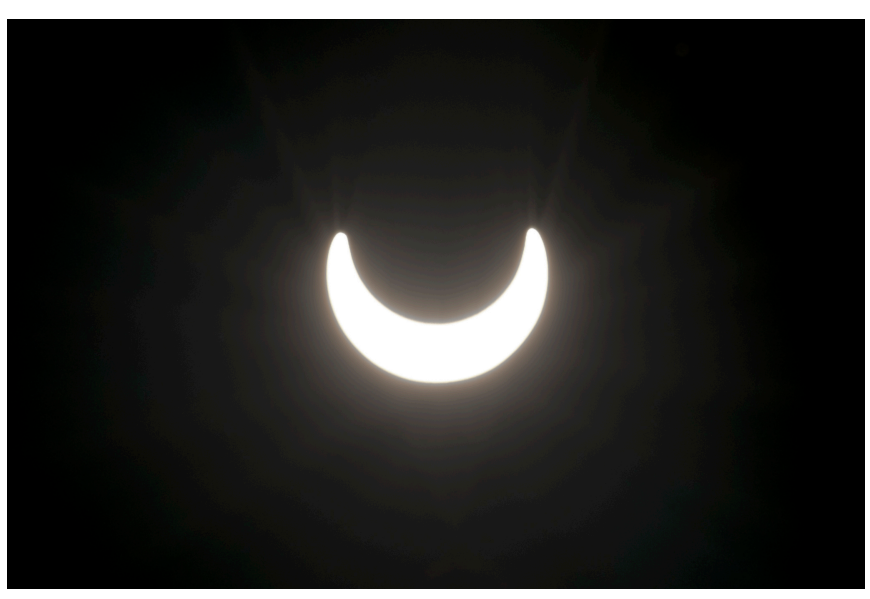

Figure 13 - Partial solar eclipse

Source: https://commons.wikimedia.org/wiki/File:Partia I_Solar_Eclipse.jpg

Permission: CC-BY-SA 3.0

Author: Ninjiangstar

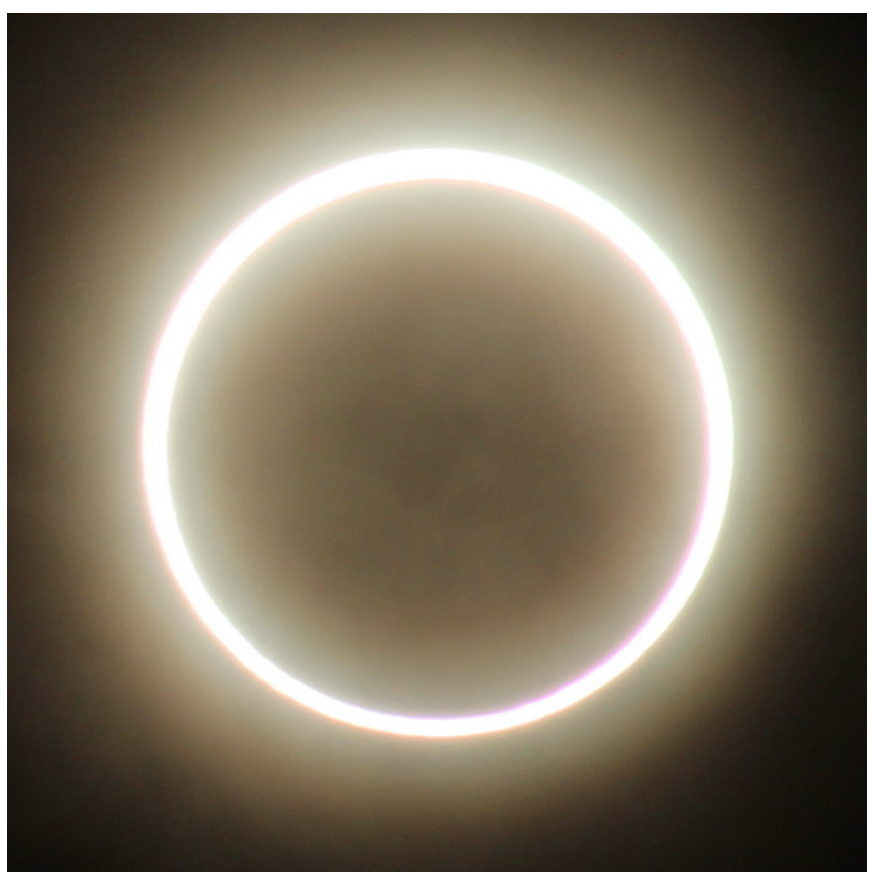

Figure 14 - Annular Phase of Solar Eclipse, May 10 2013, viewed from Churchills Head, north of Tennant Creek, Northern Territory, Australia

Source: https://commons.wikimedia.org/wiki/File: Annular_Solar_Eclipse_May_10_2013_Northern_Territo ry_Australia.JPG

Permission: CC-BY-SA 3.0

Author: Mrpulley
Since the Moon orbits Earth every 29.5 days, it seems to make sense that we should experience lunar and solar eclipses every month. However, we know that solar eclipses only occur 2-5 times annually, ${ }^{19}$ and lunar eclipses happen 2-4 times per year. ${ }^{19}$ This is because the Moon's orbit around Earth is tilted by about $5^{\circ}$, relative to Earth's orbit around the Sun. ${ }^{19,20}$

This means that the Moon spends most of the time above or below Earth's orbit, and so the three objects do not achieve the perfect alignment that is necessary for eclipses. These perfect alignments can only occur during the period of time in which the Sun is close enough to one of the Moon's nodes-where the orbit of the Moon intersects the ecliptic. These seasons last about 31 to 37 days, and they occur about every 6 months. ${ }^{21}$ If there is a full moon during this period, a lunar eclipse will occur. If there is a new moon during the period, then a solar eclipse will occur. Since it is about 15 days between full moon and new moon, there is enough time for 2-3 eclipses during each season. ${ }^{20}$

\section{Advances and Future Impacts of Lunar Exploration}

As stated in the outset of this report, human fascination with the Moon had been stimulated by the collective effort of humanity to reach the Earth's natural satellite. Although this fascination has largely dwindled after completing our goals, the technological advances, space related discoveries, and other benefits are daily reminders of the tangible rewards of space exploration and study of astronomy for humans.

We will first look at some past explorations and present exploits from space travel to the Moon to identify some of the social benefits that have accrued over time, as well as future benefits or uses for the Moon that have yet to be evoked or finalized.

\section{Past \& Present}

It was clear historical astronomers "knew the effects of tides and other biological cycles were from the waxing and waning of the Moon, ${ }^{\prime 22}$ but it took the beginning of "space-age exploration to show us how the Moon is connected to human existence on a very fundamental level.."22

It all started with Russia's launch of Sputnik 1 in October of 1957. It became the first man-made object to be intentionally launched into space and succeed in entering Earth's orbit. From this success, it became apparent that the Moon was a reachable destination not only for probes and robotics, but potentially also for manned exploration. The repeated success of Russian robotics missions spurred other countries, mainly the United States; to pursue similar 
feats in the industry and field of study. ${ }^{22}$ Thus born in the US were the Ranger, Surveyor, and most importantly, Apollo series of missions that would all together greatly increase our knowledge of the Moon's composition and physical landscape. Information and knowledge acquired through robotic means in Ranger and Surveyor missions would then be applied to ensure the safety of human exploration in the Apollo missions. ${ }^{22}$ These initial exploratory searches completed by robotic means were vital to the success of manned missions. Additionally, the continual competition between American and Russian programs was also vital in accelerating progress. Within a decade, both programs were progressing from zero objects in space, to humans landing on the Moon. ${ }^{22}$

The next logical step was to successfully land humans on the Moon. This was accomplished in the Apollo missions, when Apollo 11 in 1969, manned by the famous crew of Neil Armstrong, Michael Collins, and Buzz Aldrin successfully landed and walked on the moon. ${ }^{22}$ It was from these missions that the first physical samples of lunar rock were brought back to the Earth to be tested and learned from. At this time, these samples along with samples collected from robotic missions, had profoundly invoked "scientists [to develop] a new view of the origin and evolution of the planets and life on Earth." ${ }^{22}$ The Apollo missions continued on and were now majorly functioning with greater focus on science and discovery. With each new Apollo mission exploring different areas on the lunar rock to develop a more complete understanding of astrophysical history and lunar composition. ${ }^{22}$ Aside from providing a strong knowledge base on lunar history, the Apollo findings were also very applicable to understanding our own planet's composition. The importance of these findings was amplified by the difficulty of studying Earth's naturally active geologic crust and core.

These early days of space exploration are decades in the past, but there are benefits created from the pursuit of space in the 6o's and 70's that have been highly applicable and advantageous to daily human lives to this day. These numerous space-inspired technologies that have made the transition to serve daily needs are continually promoted by space programs like NASA. ${ }^{23}$ There are examples of tech and science across all industries that have transcended space use; this includes products in health and medicine, transportation, public safety, consumer products, environmental resources, and more. Products within these categories can be exemplified by LEDs, artificial limbs, antiice systems, improved tires, landmine detection, fire protection, memory foam, freeze-dry technologies, software, programs, various food safety devices, water purification devices, and many more. ${ }^{23}$

A lot of daily products, unknown to the average individual, were invented for space use well before becoming commercialized for public use. Although the initial and primary objectives of first space exploration programs were to discover extraterrestrial phenomena, the programs unintentionally developed hundreds and thousands of designs and concepts that became even more applicable on Earth. This quote sums up this whole notion quite well, as "[all these different products] that made man's step on the Moon possible soon led to giant leaps in technology on Earth. ${ }^{24}$

\section{Future}

The interest in future space exploration and space programs like NASA have dwindled since the expedition of the Moon. However, there are ideas, lessons, and certainly assets that can be utilized as learning experiences and directives for future missions.

It is quite clear that previously space missions and exploration of the Moon was largely driven by curiosity and to simply prove it was possible. However, NASA now believes if any return to the moon were to occur it would be to discover new ways the Moon can help grow and support spacefaring capabilities, as well as practice and develop skills and technologies that can be applied to other worlds. ${ }^{22}$ At this point and time, NASA has little motivation to send manned units to return to the Moon. It is largely understood what the lunar rock has to offer, and that any missions returning to the Moon are largely to "orbit the moon, [and make] global maps of unsurpassed quality. ${ }^{\prime 22}$ It becomes apparent the Moon has been marked basically as a testing ground for new and future technologies that NASA is testing for further space exploration. Examining a recent press release from NASA, their interests lie in a variety of categories that do not include the Moon. Instead, key points regarding the Journey to Mars, the ISS, technological advancement, the Earth, and beyond our solar system were the more pertinent issues. ${ }^{25}$ To that point, the Moon and its relevance to Earthly matters are not done or over. Instead, the success and lessons learned from human's first endeavours into space are continually applied to future space programs. This is clear in the press release that although the Moon is not a headlined topic, it was the curiosity, exploration, and results of exploring the Moon that has now motivated astronomers, astronauts, and scientists to seek bigger and more impressive goals.

\section{Acknowledgments}

We would like to thank Dr. Daryl Janzen for his expertise, dedication and contagious enthusiasm in teaching "Astronomy of Planets," for which this paper was written. 


\section{References}

${ }^{1}$ USA Government, What Causes

Tides? http://scijinks.jpl.nasa.gov/tides/ (Accessed 2017)

${ }^{2}$ P. Henney, How Earth and the Moon Interact, http://www.astronomytoday.com/astronomy/earthmoon.h tml (Accessed 2017)

${ }^{3}$ R. Gigli, Galileo's Theory of the Tides, http://galileo.rice.edu/sci/observations/tides.html (Accessed 2017)

${ }^{4}$ M. Ekman, A Concise History of the Theories of Tides, Precession-Nutation and Polar Motion (From Antiquity to 1950), http://www.afhalifax.ca/magazine/wpcontent/sciences/vignettes/supernova/nature/MAREES/Hist oireMarees.pdf (1993)

${ }^{5}$ K.E. Carr, What is Inertia? Quatr.us Study Guides, http://quatr.us/physics/space/inertia.htm\#topbar (2016)

${ }^{6} \mathrm{D}$. Byrd, Tides, and the pull of the moon and sun, http://Earthsky.org/Earth/tides-and-the-pull-of-themoon-and-sun\#two-tides (2016)

${ }^{7}$ MoonConnection.com, Understanding The Moon Phases, http://www.moonconnection.com/moon_phases.p html (2017)

${ }^{8}$ J. Dejoie and E. Truelove, What are the Phases of the Moon? High Energy Astrophysics Science Archive Research Center, Astrophysics Science Division, National Aeronautics and Space Administration/Goddard Space Flight Center https://starchild.gsfc.nasa.gov/docs/StarChild/questi ons/question3.html (Accessed 2017)

${ }^{9}$ C. Choi, How the Moon Formed: Violent Cosmic Crash Theory Gets Double Boost, http://www.space.com/29047how-moon-formed-earth-collision-theory.html (2015)

$10 \mathrm{~J}$. Villanueva, Milankovich

Cycle, http://www.universetoday.com/39012/milankovitchcycle (2015)

${ }^{11}$ C. Choi, Moon Facts: Fun Information About the Earth's Moon, http://www.space.com/55-earths-moon-formationcomposition-and-orbit.html (2014)

${ }^{12}$ N. T. Redd, Earth's Stabilizing Moon May Be Unique Within Universe, http://www.space.com/12464-earthmoon-unique-solar-system-universe.html (2011)
${ }^{13}$ NASA Earth Observatory, Milutan Milankovich (18791958), http://earthobservatory.nasa.gov/Features/Milankovi tch/milankovitch_2.php (2016)

${ }^{14} \mathrm{E}$. McLamb, The Tilting of the Earth: Shaping Our Seasons and Climates, http://www.ecology.com/2011/og/10/tiltingearth-shaping-seasons/ (2011)

${ }^{15}$ Swinburne University, COSMOS: The SAO Encyclopedia of Astronomy - Solstice, http://astronomy.swin.edu.au/cms/astro/cosmos/*/Solstice (2016)

${ }^{16}$ Planetary Science Institute, FAO Earth \& Moon, https://www.psi.edu/epo/faq/earth_moon.html (Accessed 2017)

${ }^{17}$ Astronomy 101, Lunar Libration: How much of the Moon's surface can we see from Earth? Skywise Unlimited https://www.wwu.edu/depts/skywise/lunar_libration.html (Accessed 2017)

${ }^{18}$ National Aeronautics and Space Administration, Moon Phase and Libration, 2016, Scientific Visualization Studio, https://svs.gsfc.nasa.gov/4404 (2015)

${ }^{19}$ F. Espenak, Lunar Eclipses for Beginners, http://mreclipse.com/Special/LEprimer.html (2014)

${ }^{20}$ F. Espenak, Solar Eclipses for Beginners, http://mreclipse.com/Special/SEprimer.html (2014)

${ }^{21}$ OMICS International Open Access Journals, OMICS Publishing Group, Eclipse Season, http://research.omicsgroup.org/index.php/Eclipse_season ( 2014)

${ }^{22}$ P. Spudis, Lunar Exploration: Past and Future, https://www.nasa.gov/50th/50th_magazine/lunarEx ploration.html (2008)

${ }^{23}$ NASA, NASA Technologies Benefit Our Lives, https://spinoff.nasa.gov/Spinoff2008/tech_benefits.h tml (2009)

${ }^{24}$ NASA, NASA Facts: Lyndon B. Johnson Space Center, https://www.nasa.gov/sites/default/files/8o66omain _ApolloFS.pdf (2004)

${ }^{25}$ NASA, What's next for NASA?, https://www.nasa.gov/about/whats_next.html (2016) 
Earth and the Moon (Finney et al.)

University of Saskatchewan Undegraduate Research Journal 